\title{
Acknowledgment
}

The editor acknowledges the ad hoc reviewers who reviewed manuscripts for The Humanistic Psychologist between October 2018 and September 2019 and thanks them for their time and effort.

Guy Albert

Kyle Arnold

Alice Chirico

David Coghlan

Ross Crisp

Helena Dahlberg

Virginia Eatough

Andrew J. Felder

Jessie Goicoechea
Daniel A. Helminiak Edward Hoffman

Robert Kugelmann

Leswin Laubscher

Claire LeBeau

Rebecca Lloyd

Doug MacDonald

Geoffrey Charles Manzi

Donald Moss

Elisabeth Punzi

\section{Peter Rosan}

Seth Segall

Stephen Smith

Drake Spaeth

Stephanie S. Swales

Russell Walsh

Simon Wharne

Rachel Lev Wiesel

Christine N. Winston 